\title{
Perfiles motivacionales, estrategias volitivas y rendimiento académico en ciencias exactas y experimentales de bachillerato
}

\section{María del Socorro Rodríguez Guardado, Martha Leticia Gaeta González ${ }^{1}$}

\footnotetext{
${ }^{1}$ Facultad de Educación, Universidad Popular Autónoma del Estado de Puebla, México
}

\section{México}




\section{Resumen}

Introducción. En el Bachillerato, las asignaturas de ciencias exactas y experimentales demandan el desarrollo de competencia científica, que a su vez requiere el dominio del conocimiento teórico y del conocimiento práctico, pero también de la motivación y el esfuerzo sostenido para un mayor involucramiento y desempeño académico. De ahí la relevancia de conocer la diversidad motivacional que orienta al alumnado a involucrarse (o no) en el aprendizaje de las ciencias, así como las estrategias necesarias para que perseveren en el logro de sus metas, a partir de sus propios intereses. El objetivo de esta investigación fue analizar los perfiles motivacionales de los estudiantes y valorar las posibles relaciones con las estrategias volitivas y el rendimiento académico en las asignaturas de ciencias experimentales y exactas en el Nivel Medio Superior.

Método. El presente estudio se llevó a cabo mediante un diseño cuantitativo no experimental transversal. Participaron 204 estudiantes de cuatro bachilleratos, con edades comprendidas entre los 16 y 18 años, quienes respondieron el Inventario de Estrategias Volitivas (AVSI) y el Cuestionario de Metas Académicas (CMA), previamente validados.

Resultados. Los resultados mostraron que el uso diferenciado de estrategias volitivas está relacionado con el perfil motivacional de los estudiantes. Las estrategias volitivas de control de la autoeficacia se relacionan significativamente con el rendimiento académico.

Discusión y conclusiones. Este trabajo evidencia la necesidad de promover estrategias motivacionales y emocionales, además de las cognitivas, para un mejor aprendizaje en el Nivel Medio Superior. Asimismo, es importante considerar incluir en el curriculum el fortalecimiento de las estrategias volitivas para que los estudiantes persistan ante las dificultades y se mantengan motivados para lograr sus metas académicas.

Palabras Clave: Aprendizaje autorregulado, motivación, voluntad, bachillerato, rendimiento académico. 


\begin{abstract}
Introduction. In upper high school, exact and experimental sciences subjects demand the development of scientific competence, which in turn requires mastery of theoretical and practical knowledge, but also motivation and sustained effort for greater academic involvement and performance. Hence it is necessary to know the motivational diversity that guides students to get involved (or not) in the learning of science, as well as the necessary strategies to persevere in achieving their goals based on their interests. The objective of this research was to analyze the motivational profiles of the students and assess the possible relationships with volitional strategies and academic performance in the subjects of experimental and exact sciences in Upper High School.
\end{abstract}

Method. The present study was carried out through a cross-sectional non-experimental quantitative design. Participants were 204 students, aged between 16 and 18, from four high schools, who answered the Volitional Strategies Inventory (AVSI) and the Academic Goals Questionnaire (CMA), previously validated.

Results. Results showed that the differentiated use of volitional strategies is related to the motivational profile of the students. Volitional self-efficacy control strategies are significantly related to academic performance.

Discussion and Conclusions. This work demonstrates the need to promote motivational and emotional strategies, in addition to cognitive ones, for better learning at Upper High School. It is also important to consider including in the curriculum the strengthening of volitional strategies so that students persist in the face of difficulties and remain motivated to achieve their academic goals.

Keywords: Self-regulated learning, motivation, volition, academic performance. 


\section{Introducción}

Saber observar, experimentar y comunicar lo que se aprende y se investiga son actividades que deben desarrollar quienes estudian ciencias exactas y experimentales. Sin embargo, la enseñanza de la ciencia no es tarea fácil, y promover el interés en lo jóvenes hacia las actividades o trabajos que requiere el aprendizaje de las ciencias siempre ha sido un reto para los educadores. La problemática puede atribuirse a la baja motivación y al poco esfuerzo sostenido de los estudiantes.

Durante muchos años, la mayor parte de los estudios sobre el aprendizaje de las ciencias ha tenido que ver con las dificultades conceptuales o procedimentales del alumnado (Campanario y Otero, 2000), habiendo muy poca investigación sobre sus motivaciones y las estrategias que utilizan para un mayor involucramiento y desempeño académico. De ahí que conocer lo que motiva y orienta a los estudiantes a involucrarse (o no) en el aprendizaje de las ciencias es relevante, así como atender a la diversidad motivacional para que alcancen sus metas y éxito académico a partir de sus intereses personales.

En tal sentido, la teoría de las metas de logro constituye una de las líneas de investigación importantes para explicar la motivación académica (Gaeta, 2009). Los avances y aportes recientes de diferentes investigaciones en relación con las metas académicas se han centrado principalmente en la distinción entre diferentes orientaciones a metas (Alemán, Trías y Curione, 2011; De la Fuente, Pichardo, Justicia y Berbén, 2008; Martín, Bueno y Ramínez, 2010; Navas, Soriano, Holgado y Jover, 2016), siendo pocos los trabajos que han abordado su estudio a partir de diferentes combinaciones de metas - los perfiles motivacionales de los estudiantes (Valle et al., 2006; Valle et al., 2015) o los que han considerado el uso de las estrategias que permitan mantener la atención y el esfuerzo en estos procesos - estrategias volitivas (Bartels, Magun-Jackson y Kemp, 2009; Broc, 2015; Gaeta, Teruel y Orejudo, 2012; McCann y Turner, 2006; Valle, Cabanach, Rodríguez, Núñez y González-Pienda, 2006).

Tomando en consideración que estos y otros esfuerzos investigativos realizados a la fecha no han analizado estos aspectos de manera conjunta, en el presente estudio nos propusimos analizar los perfiles motivacionales, el uso de estrategias volitivas y el rendimiento académico de los estudiantes de bachillerato. Consideramos relevante su estudio en las asignaturas de ciencias exactas y experimentales, cuyos contenidos demandan el desarrollo de 
competencia científica que a su vez requiere el dominio del conocimiento teórico, del conocimiento práctico y de la orientación motivacional. La OCDE (2006) refiere que estos dominios deben incluir la identificación de problemáticas, la adquisición de nuevos conocimientos, la explicación de los fenómenos científicos y la extracción de conclusiones, así como el uso y análisis de textos para emitir juicios fundamentados. Al respecto, Franco (2015) agrupa en siete dimensiones las competencias científicas: planificación y diseño, recolección y procesamiento de datos, el análisis, las conclusiones, reflexión crítica y la comunicación de los resultados obtenidos y la actitud para trabajar en equipo. Todo ello hace necesario el fortalecimiento de aspectos motivacionales y volitivos por parte del alumnado.

\section{Motivación y volición}

La motivación ha sido considerada como un constructo hipotético que explica el inicio y dirección de una conducta hacia una meta (De la Fuente, 2004). Así, la motivación responde la pregunta ¿hacia dónde y hacia qué está dirigida la energía o el movimiento? (Pintrich, 2003). De ahí que la motivación es responsable en gran medida de que continuemos realizando una actividad, es un estado interno que nos anima a actuar, nos dirige en determinadas direcciones y nos mantiene en algunas actividades (Ormrod, 2005).

En este sentido, tradicionalmente la mayoría de los estudios se han centrado en dos orientaciones de meta: al aprendizaje y al rendimiento. Sin embargo, en años más recientes, el estudio de la diversidad de metas ha tenido mayor preponderancia de modo que los modelos normativos han establecido metas académicas divididas en metas de aprendizaje o maestría, metas de ejecución, actuación o rendimiento, metas de evitación, metas centradas en el yo y metas sociales (Midgley et al., 2001; Wentzel, 2001).

Los trabajos de Pintrich (2000) evidencian la posibilidad de metas múltiples, es decir, los estudiantes pueden adoptar distintas metas de manera complementaria, presentándose una interacción entre metas de aprendizaje y rendimiento para diferentes motivaciones o resultados cognitivos. Con respecto a la combinación de metas, Pintrich (2000) agrega el factor social, asumiendo que en un salón de clase se presentan situaciones de competencia y comparación social, y los estudiantes pueden adoptar diferentes combinaciones de metas, aplicando múltiples patrones de metas que pueden seguir distintas estrategias regulatorias. 
La gestión de metas en los jóvenes para lograr sus objetivos da lugar a diferentes perfiles motivacionales. Así pues, estudiar las distintas orientaciones a metas académicas desde esta perspectiva es uno de los propósitos que nos planteamos en este estudio, considerando que cada perfil representa una combinación determinada de motivos en los estudiantes, pero también da una manera distinta de estar motivado a nivel académico.

Los perfiles motivacionales contemplan distintas orientaciones a metas dentro de un mismo sujeto porque puede estar motivado por diferentes razones. Así los estudiantes pueden mostrar interés y satisfacción por el estudio, en cuyo caso tendrán orientación hacia metas de aprendizaje. Por su parte, los estudiantes que adoptan una perspectiva de comparación, es decir, que no quieren parecer incompetentes y constantemente se miden con lo que hacen otros compañeros muy probablemente presenten metas de logro o evitación. Por otro lado, la necesidad del reconocimiento ante profesores, compañeros y familiares son características que definen las metas de refuerzo social, que también pueden estar presentes junto con la meta de aprendizaje o de comparación (Pintrich, 1999; Pintrich, 2000).

Por otro lado, en el ámbito escolar, la voluntad para mantener la motivación juega un papel fundamental (Gaeta, Cavazos, Sánchez, Rosário y Högemann, 2015), sobre todo cuando las tareas y proyectos requieren de un tiempo y esfuerzo mayores para completarse. En este sentido, los modelos sobre el aprendizaje autorregulado consideran el control autorregulatorio o volitivo, que implica mantener la intención y el esfuerzo para involucrarse o completar las actividades. El control volitivo involucra el uso de estrategias dirigidas a la regulación de emociones, motivación y cognición en el proceso de esfuerzo a la meta (Corno, 1993; Corno y Kanfer, 1993; Kuhl, 1987). Se trata de un constructo que tiene un impacto directo sobre el comportamiento de logro, ya que media entre la intención para aprender y el esfuerzo por lograr alcanzar la meta establecida.

La volición es, por lo tanto, un soporte importante en los aspectos de la cognición tales como la profundización del proceso que, a su vez, refleja estrategias de automotivación y de control en el que las emociones inapropiadas se asocian con ineficacia o impotencia (Corno, 1993). Incluso los estudiantes comprometidos pueden distraerse de las metas propuestas, por lo que las estrategias volitivas cobran importancia al proteger las intenciones de los educandos por aprender, de distracciones internas y externas, y al mantener la concentración y el esfuerzo. Las estrategias volitivas controlan y dirigen el procesamiento de la infor- 
mación, los afectos y las conductas que llevan a la realización de los objetivos académicos. Así los procesos volitivos alientan las acciones y fortalecen los aspectos motivacionales (Corno, 1993; McCann y García, 1999; McCann y Turner, 2004).

Las estrategias volitivas se pueden definir como los pensamientos y/o comportamientos que son dirigidos para mantener la intención y lograr una meta propuesta a pesar de las distracciones y obstáculos de diferente índole que se puedan presentar, estas juegan un papel fundamental en la intención por aprender y mantener la motivación. Esta intención por aprender se podría ver reflejada en la obtención de buenas calificaciones en las evaluaciones y en las tareas escolares, viéndose reflejado un rendimiento académico satisfactorio.

Como puede observarse la motivación y la volición son conceptos diferentes, sin embargo, no pueden separase porque están implícitos en la autorregulación de los estudiantes para lograr las metas y alcanzar un rendimiento académico satisfactorio. Los educandos necesitan conocer cómo llevar acabo esta unión porque al motivarse para establecer sus metas deben considerar los hábitos de trabajo, y desde luego, el manejo de las estrategias volitivas que les permitirán persistir hasta su cumplimiento.

\section{Objetivos e hipótesis}

Con base en lo indicado previamente, el objetivo principal que se propone es: analizar los perfiles motivacionales de los estudiantes y valorar las posibles relaciones con las estrategias volitivas y el rendimiento académico en las asignaturas de ciencias experimentales y exactas en el Nivel Medio Superior.

Desde el objetivo propuesto se formula la siguiente hipótesis: el uso de estrategias volitivas y el rendimiento académico varían de acuerdo con el perfil motivacional de los estudiaintes de ciencias exactas y experimentales en el Nivel Medio Superior (Bachillerato).

Con este trabajo esperamos poder aportar evidencia sobre la variedad de metas que los estudiantes establecen (perfiles motivacionales) y que permiten (o no) hacer uso de diferentes estrategias volitivas para perseverar en el estudio y obtener un rendimiento académico satisfactorio. 


\section{Método}

\section{Participantes}

Los estudiantes objeto de estudio pertenecen a cuatro centros educativos ubicados en la ciudad de Puebla, México, seleccionados por accesibilidad. La muestra total está integrada por 204 estudiantes matriculados en el cuarto semestre de Educación Media Superior (Bachillerato), 130 mujeres (63.7\%) y 74 hombres (36.3\%), con edades comprendidas entre los 16 y 18 años $(M=16.55 ; D T=.95)$. El número de estudiantes de escuelas públicas fue de 134 $(65.7 \%)$ y de escuelas privadas de $70(34.3 \%)$.

\section{Instrumentos}

Para identificar el uso de estrategias volitivas por los estudiantes se utilizó el Inventario de Estrategias Volitivas Académicas (AVSI) propuesto por McCann y García (1999) y traducido al español por Gaeta (2009). Autoinforme que mide las tendencias de los educandos hacia el control volitivo en contextos académicos. Este cuestionario considera tres factores: autoeficacia (ej. "Me digo: puedes hacerlo"), reducción del estrés (ej. "A menudo busco usar algún método de relajación para concentrarme mejor en mis estudios") e incentivos base negativa (ej."Pienso en lo decepcionados que van a estar mis familiares y amigos si repruebo").

El instrumento original contiene 20 ítems medidos por una escala Likert de cinco puntos que oscila desde "Muy en desacuerdo" (1) hasta "Muy de acuerdo" (5). La consistencia interna reporta un (alfa de Cronbach) es de .82 para la autoeficacia, .69 para la reducción del estrés y .87 para incentivos base negativa, con un valor alpha para la escala total de .87 (McCann y García, 1999; McCann y Turner, 2004).

El Achievement Goals Tendencies Questionnaire, propuesto por Hayamizu y Weiner (1991) identifica la tendencia del individuo a tratar de superar obstáculos y avanzar para lograr lo que desea. La versión adaptada al español, Cuestionario de Metas Académicas (CMA), de Núñez, González-Pienda, González-Pumariega, García y Roces (1997), distingue cuatro tipos de metas: con orientación al aprendizaje (ej. "Me gusta conocer cosas nuevas"), con orientación al yo (ej. "Quiero que la gente vea lo inteligente que soy”), con orientación a la valoración social (ej. "No quiero que mis compañeros se burlen de mi”) y con orientación al logro (ej. "Quiero ser valorado por mis amigos"). El instrumento original, de 20 ítems, obtuvo 
una consistencia interna total de .88 y utiliza escala de respuesta Likert. Las propiedades psicométricas del CMA fueron examinadas por Gaeta et al. (2015) con estudiantes universitarios mexicanos, obteniendo una consistencia interna que osciló entre .78 y .89. En este trabajo el CMA mostró un valor alpha de .814 .

El rendimiento académico se consideró como el promedio de las calificaciones reportadas por los educandos en las asignaturas de matemáticas, física y química, obtenidas hasta el cuarto semestre del Bachillerato. Asimismo, se consideraron las asignaturas reprobadas al momento de la aplicación del instrumento.

\section{Procedimiento}

Se seleccionó el cuarto semestre de Bachillerato porque se considera que el alumnado ya ha pasado el proceso de adaptación que este nivel educativo exige. Además, de acuerdo con Sanz de Acedo, Ugarte y Lumbreras (2003) en el estudio que realizaron sobre la importancia que los adolescentes en edades de entre los 15 y 19 años otorgan a las metas, mostraron que en esta etapa los estudiantes reafirman la necesidad de mostrar su imagen y valía ante los iguales y en consecuencia conseguir reputación y los buenos resultados académicos apoyan la imagen social, por lo que una diversidad de metas puede estar presente en esta etapa.

Los permisos necesarios para aplicar el instrumento (a los directivos, profesores de asignatura y padres de familia), se solicitaron de manera previa a la aplicación dado que los participantes son menores de edad. La aplicación en cada uno de los centros se realizó de manera colectiva dentro del horario escolar; el tiempo promedio de respuesta fue de 20 minutos. La participación de los estudiantes fue voluntaria y se respetó la confidencialidad de sus datos personales y de las respuestas al instrumento.

\section{Análisis de datos}

Después de determinar las propiedades psicométricas de los instrumentos, se realizó un análisis de clusters o conglomerados para profundizar en el conocimiento de los perfiles motivacionales de los estudiantes; se determinó la factibilidad de los grupos mediante conglomerados jerárquicos y posteriormente se efectuó el análisis de conglomerados K-medias. Una vez identificados los perfiles motivacionales de los educandos se llevó a cabo una prueba Kruskal-Wallis. A continuación, se realizó la prueba Mann-Whitney-Wilcoxon para analizar la heterogeneidad en los perfiles motivacionales, con respecto al uso de las estrategias voliti- 
vas. Finalmente se realizó una regresión para analizar la incidencia de las estrategias volitivas en el rendimiento académico. Para los análisis estadísticos se utilizó el programa SPSS versión 22.0 .

\section{Resultados}

De los cuatro perfiles motivacionales de los estudiantes participantes, el primero, integrado por 58 estudiantes, correspondió a la orientación a las metas de logro; el segundo, con 81 participantes, de orientación a múltiples metas; en el tercero se ubicaron 17 estudiantes sin ninguna orientación y; en el cuarto conglomerado se ubicaron 48 estudiantes orientados al rendimiento y al aprendizaje. La figura 1 muestra los perfiles motivacionales encontrados en los estudiantes, así como las orientaciones de metas mostradas.

En cuanto a las estrategias volitivas utilizadas por los estudiantes, en primer lugar, se encuentran las de control de la autoeficacia $(M=35.09 ; D E=5.36)$, seguida por las de incentivos de base negativa $(M=18.01 ; D E=3.78)$, siendo las de reducción del estrés las menos empleadas $(M=16.31 ; D E=4.26)$.

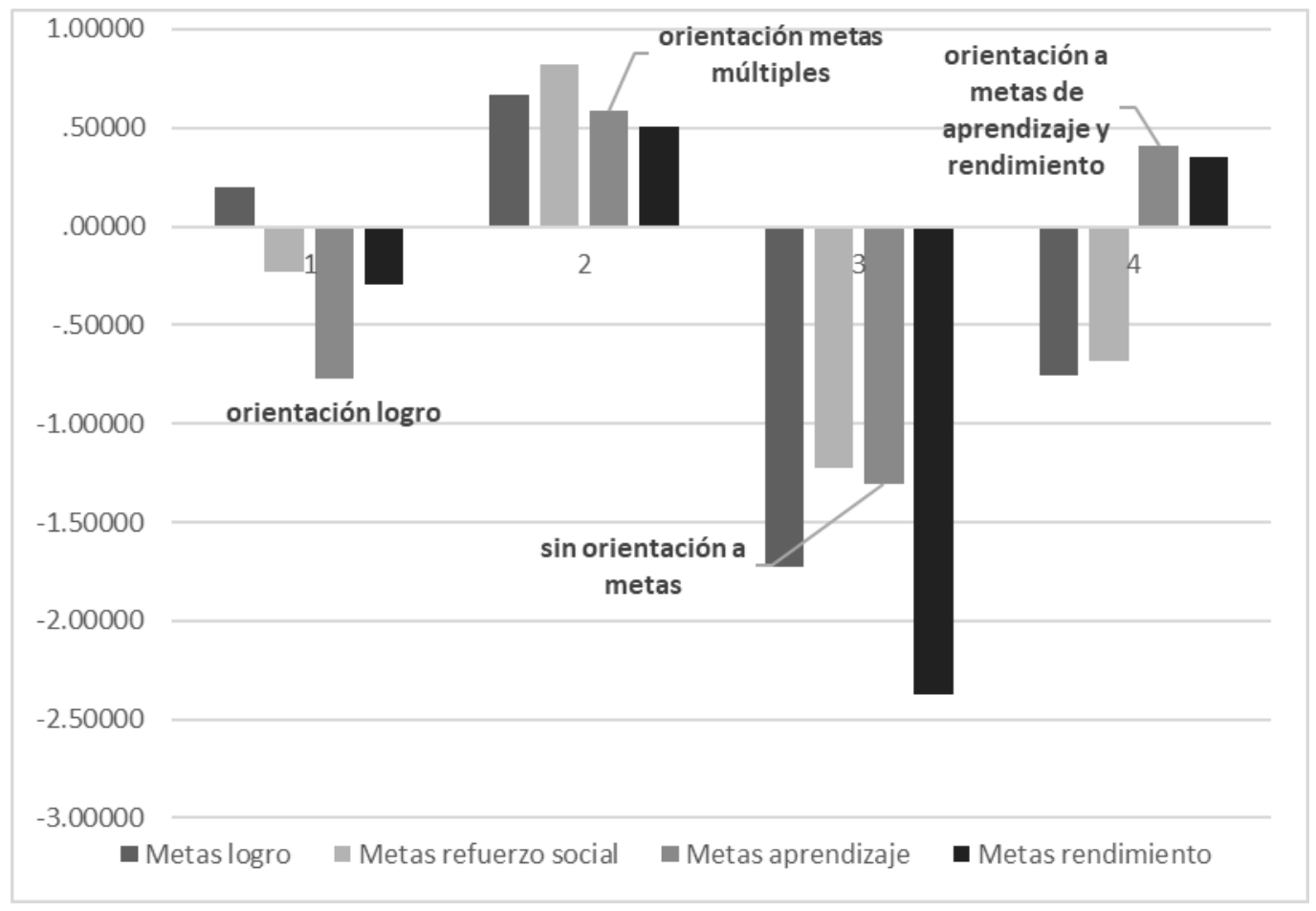

Figura 1. Perfiles motivacionales de los estudiantes. 
A partir de la prueba Kruskal-Wallis, se observó con respecto al uso de estrategias de control de la autoeficacia, que los estudiantes con perfil orientado a metas múltiples tuvieron el promedio más alto (rango promedio $=123.20$ ), seguido por los estudiantes con metas de aprendizaje y rendimiento (rango promedio $=93.95)$ y metas de logo (rango promedio $=$ 90.51) y aquellos con un perfil sin orientación a metas mostraron el valor más bajo (rango promedio $=68.94)$.

Con respecto a los incentivos de base negativa, los estudiantes con perfil orientado al rendimiento y aprendizaje (rango promedio $=79.18$ ) y los que tienen un perfil sin orientación a metas (rango promedio $=79.53$ ) reportaron valores cercanos. Los estudiantes con perfiles orientados a metas de logro obtuvieron un promedio (rango promedio $=98.88$ ) por debajo de los estudiantes con perfiles orientados a metas múltiples (rango promedio $=123.73$ ).

En cuanto a la reducción del estrés, los estudiantes con perfiles orientados al logro y aquellos con orientación a metas de rendimiento y aprendizaje tuvieron un promedio similar (93.47 y 93.21 respectivamente), mientras que los que tienen un perfil sin orientación a metas el rango promedio fue de 75.44 y los que tiene un perfil orientado a múltiples metas el valor fue de 120.15 .

Se realizó la prueba Mann-Whitney-Wilcoxon para analizar la heterogeneidad en los perfiles motivacionales de los estudaintes, con respecto al uso de las estrategias volitivas. Al comparar a los estudiantes con perfil orientado a metas de logro con el de orientación a metas múltiples, se observó una diferencia significativa en el uso de las estrategias volitivas tanto de control de la autoeficacia $(z=3.21 ; p=.00)$, en los incentivos de base negativa $(z=2.66 ; p=$ $.01)$ y en la reducción del estrés $(z=2.63 ; p=.01)$.

La comparación entre el perfil motivacional con orientación a metas múltiples y el perfil que no presentó ninguna orientación a metas tuvo una diferencia significativa en la autoeficacia $(z=3.08 ; p=.00)$, incentivo de base negativa $(z=2.68 ; p=.01)$ y en la reducción del estrés $(z=2.63 ; p=.01)$.

Al comparar los perfiles motivacionales de orientación a metas múltiples con los de orientación al aprendizaje y rendimiento, se observó que existen diferencias significativas en 
el control de la autoeficacia $(z=2.93 ; p=.00)$, los incentivos de base negativa $(z=3.97 ; p$ $=.00)$ y la reducción del estrés $(z=2.63 ; p=.01)$.

A partir del análisis de regresión lineal múltiple de tipo jerárquico (considerando como variable predictora a las estrategias volitivas y como variable criterio al rendimiento académico) se observó que las estrategias volitivas totales no influyen significativamente sobre el rendimiento académico $(F=1.62 ; p=.19)$, pero un análisis más detallado mostró que las estrategias de control de la autoeficacia si lo hacen, aunque el tamaño del efecto es pequeño $\left(\beta=.21 ; p=.03, R^{2}=.024\right)$. La tabla 1 muetra estos resultados.

Tabla 1. Resultados del análisis de regresión.

\begin{tabular}{|c|c|c|c|c|c|}
\hline \multirow[b]{2}{*}{ Modelo } & \multicolumn{2}{|c|}{$\begin{array}{l}\text { Coeficientes no estandariza- } \\
\text { dos }\end{array}$} & \multirow{2}{*}{$\begin{array}{c}\text { Coeficientes } \\
\text { estandarizados } \\
\text { Beta }\end{array}$} & \multirow[b]{2}{*}{$t$} & \multirow[b]{2}{*}{$p$} \\
\hline & $B$ & Error estándar & & & \\
\hline (Constante) & 7.927 & .477 & & 16.633 & .000 \\
\hline AUTOEFICACIA & .039 & .018 & .212 & 2.170 & .031 \\
\hline INBANEG & -.007 & .019 & -.032 & -.391 & .696 \\
\hline REDESTRES & -.032 & .025 & -.118 & -1.281 & .202 \\
\hline
\end{tabular}

a. Variable dependiente: Rendimiento académico

Nota. INBANEG = Incentivos de base negativa. REDESTRES = Reducción del estrés.

\section{Discusión y conclusiones}

Este estudio tuvo como objetivo principal analizar los perfiles motivacionales de los estudiantes y valorar las posibles relaciones con las estrategias volitivas y el rendimiento académico en las asignaturas de ciencias experimentales y exactas en el Nivel Medio Superior.

Los resultados mostraron la existencia de cuatro perfiles motivacionales en los estudiantes: perfil orientado a metas de logro; perfil con orientación a metas múltiples (aprendizaje, rendimiento, logro y refuerzo social); perfil sin orientación a ninguna meta y perfil motivacional integrado por metas de aprendizaje y de rendimiento. 
A partir de estos hallazgos se corroboró la posibilidad de que en los estudiantes coexistan varios tipos de metas; así un estudiante puede buscar orientarse tanto a la obtención de un buen rendimiento académico a la vez que busca mejorar su aprendizaje, hacer amigos o buscar un reconocimiento (metas sociales) o también puede proponerse una meta de logro para obtener recompensas o premios. De esta forma, los estudiantes se orientan a varias metas para aproximarse a la tarea, lo que concuerda con otras investigaciones previas (Pintrich, 2000; Navas y Sampascual, 2008; González, Valle, Suárez y Fernándes, 1999).

De manera más específica, la mayor parte de los estudiantes $(n=81)$, mostraron tener un perfil con orientación a metas múltiples, en el cual se utilizaron mayormente las metas de refuerzo social. Tal y como refieren Juvonen, Wentzel y Gutiérrez (2001) la búsqueda de metas sociales se relaciona positivamente con la motivación académica y el desempeño, por lo que en este caso puede ser un aliciente para los estudiantes para involucrarse en el estudio.

Por otro lado, respecto al uso de estrategias volitivas, los resultados mostraron que los estudiantes hacen un mayor uso de estrategias volitivas de tipo motivacional, como es el control de la autoeficacia. Por su parte, las evidencias mostraron que el uso de estrategias volitivas referidas a la emoción, como la reducción del estrés e incentivos base negativa fueron utilizadas con menor frecuencia por los estudiantes.

Estos resultados nos orientan a profundizar sobre las razones que llevan a los estudiantes a privilegiar estrategias de tipo motivacional, que afectivo. Consideramos que los procesos emocionales constituyen un factor relevante para explicar el aprendizaje de los estudiantes; a veces los logros que los estudiantes obtienen han requerido de esfuerzo y tiempo lo que hace que puedan caer en actitudes y estados emocionales que dificulten sus objetivos (Pintrich y Schunk, citado en Estévez, Rodríguez, Valle, Regueiro y Piñeiro 2016). Al respecto Gaeta et al. (2012) muestran además del establecimiento de metas para que el alumno se involucre en su aprendizaje, el uso de las estrategias volitivas, tanto de carácter motivacional como afectivo, es necesario para que mantenga su intención y esfuerzo.

Los resultados mostraron además que hay una tendencia diferencial a usar y generar estrategias volitivas, de acuerdo con su perfil motivacional. Tal como establecen García y Pintrich (1993), el control volitivo es una variable de diferencia individual que contribuye a mantener la motivación y el esfuerzo en un contexto determinado. Así, al identificar las estra- 
tegias volitivas en relación con los perfiles motivacionales, se observó que las de control de la autoeficacia fueron las más utilizadas por los estudiantes con perfil motivacional de metas múltiples, con perfil orientado al logro y con perfil orientado al aprendizaje y rendimiento, pero no así con el perfil sin orientación a metas. Con respecto a los incentivos base negativa los estudiantes con perfil motivacional de metas múltiples mostraron usar con mayor grado estas estrategias, sin embargo, en las estrategias comprendidas en la reducción del estrés reportaron un menor uso, lo cual orienta a profundizar en estos aspectos en futuras investigaciones.

Al analizar la incidencia del uso de estrategias volitivas totales en el rendimiento académico, se observó que ésta no es significativa. Sin embargo, un análisis más detallado mostró que las estrategias volitivas referidas al control de la autoeficacia influyen positiva y significativamente el rendimiento académico, aunque el efecto es pequeño. Los resultados coinciden en general con los hallazgos de Broc (2015) quien encontró que las estrategias volitivas podrían ser variables latentes con influencia indirecta sobre el rendimiento académico, pero directa con las estrategias de aprendizaje, concluyendo que los aprendices necesitan estrategias volitivas que les ayuden a mantener la decisión de continuar concentrados en la tarea. Con ello se observa la necesidad de promover en el aula el uso de estrategias volitivas, considerando no solo las de control de la motivación, sino aquellas estrategias que impliquen el control de las emociones (incentivos de base negativa y reducción de estrés).

Al respecto, González et al. (1999) concluyen que el aprendizaje significativo y el rendimiento académico están influenciados directamente por variables motivacionales, una de ellas la autoeficacia. Así, la autoeficacia se identifica con los juicios que realiza la persona sobre sus propias capacidades para lograr y hacer actividades para llegar a resultados lo que potencia el compromiso de logro (Schunk y Zimmerman, 2008). Así, los individuos motivados reportan mayor autoeficacia y tendencia a la acción (Bartels et al., 2009) y una creencia fuerte de autoeficacia predice altas aspiraciones académicas (Kimm y Benneckib, 2013). De ahí la relevancia de usar estrategias que permitan mantener el sentido de autoeficacia en los estudiantes al realizar una tarea o actividad académica. Por otra parte, este estudio corrobora la propuesta de Mega, Ranconi y De Beni (2014) quienes señalan que el logro académico se atribuye a los factores emocionales de regulación y de motivación. 
Como se ha señalado, las investigaciones sobre la enseñanza de las ciencias han sido numerosas y a lo largo del tiempo han surgido diferentes paradigmas educativos con los cuales se han implementado metodologías, estrategias y diferentes recursos para promover un mejor desempeño en las asignaturas de ciencias exactas y experimentales y disminuir el alto índice de reprobación. Se puede considerar a partir de los resultados obtenidos en el presente trabajo, que el conocimiento sobre aspectos volitivos y motivacionales puede orientar la práctica educativa hacia el fortalecimiento de los aspectos mencionados, de cara a la autorregulación de los aprendizajes, que puede verse reflejada, a su vez, en un rendimiento académico satisfactorio. Este estudio evidenció la importancia que tiene considerar otros aspectos, además de los cognitivos, para un mejor desempeño académico del alumnado.

La dinamización en el proceso de enseñanza-aprendizaje de las ciencias debe considerar un enfoque integral con componentes que potencien el aprendizaje autónomo y autorregulado en diferentes contextos (Asencio, 2012). Acentuar en la práctica educativa estrategias para que la motivación del alumnado se fortalezca y se mantenga es de suma importancia. Como señala González-Torres (2012), más allá del establecimiento de metas es fundamental fortalecer en los estudiantes el uso de estrategias volitivas que les ayuden a sostener el esfuerzo y la concentración en el estudio.

Las investigaciones sobre los aspectos motivacionales y afectivos es un campo en el que hay que avanzar más, a fin de contribuir al conocimiento de estos procesos y proveer a los docentes de herramientas que contribuyan a fortalecer la autorregulación del aprendizaje en los jóvenes para un mejor desempeño académico; ello en cualquier asignatura y no únicamente en las ciencias exactas y experimentales.

En futuras investigaciones se pueden abordar, de manera conjunta, el control de la motivación, volición y cognición y considerar una muestra más amplia, mediante muestreos de tipo probabilístico, e indagar en otras asignaturas diferentes a las ciencias exactas y experimentales. Estos aspectos mencionados son limitaciones que se consideran en este trabajo. Asimismo, es necesario indagar sobre los perfiles motivacionales y el uso de estrategias volitivas en otros niveles educativos como la educación básica.

Se enfatiza, a partir de nuestros hallazgos, la relevancia de que los docentes promuevan en el aula estrategias volitivas dentro de los contenidos curriculares para ayudar al alum- 
nado a obtener resultados académicos satisfactorios en los que se atiendan aspectos afectivos, motivacionales y cognitivos que contribuyan al crecimiento personal además del académico, a partir de la identificación de sus diferentes perfiles motivacionales.

\section{Referencias}

Alemán, M. J., Trías, D. y Curione, K. (2011). Orientaciones motivacionales, rendimiento académico y género en estudiantes de bachillerato. Ciencias Psicológicas, 5(2), 159-166.

Asencio, E. (2012). Una alternativa didáctica para el perfeccionamiento del proceso de enseñanza aprendizaje de las ciencias. Revista Iberoamericana de Educación, 58, 81-97.

Bartels, J. M., Magun-Jackson, S. y Kemp, D. A. (2009). Volitional regulation and self-regulated learning: an examination of individual differences in approach-avoidance achievement motivation. Electronic Journal of Research in Educational Psychology, 7(2), 605-626.

Broc, M. A. (2015). Voluntad para estudiar, regulación del esfuerzo. Revista de Investigación Educativa, 29(1), 171-185.

Campanario, J. M. y Otero, J. C. (2000). Más allá de las ideas previas como dificultades de aprendizaje: las pautas de pensamiento, las concepciones epistemológicas y las estrategias metacognitivas de los alumnos en ciencias. Revista Electrónica Enseñanza de las Ciencias, 18, 155-169.

Corno, L. (1993). The Best Laid Plans: Modern Conceptions of Volition and Educational. Educational Research Associations, 22(2), 14-22.

Corno, L. y Kanfer, R. (1993). The role of volition in learning and performance. Review of Research in Education, 19, 301-341.

De la Fuente, A. J. (2004). Perspectivas recientes en el estudio de la motivación: la teoría de la orientación a meta. Electronic Journal of Research in Educational Psychology, 2(1), 35-62. doi:10.25115/ejrep.3.121.

De la Fuente, A. J., Pichardo, M. C., Justicia, F. y Berbén, A. (2008). Enfoques de aprendizaje, autorregulación y rendimiento en tres universidades europeas. Psicothema, 20(4), 705-511.

Estévez, I., Rodríguez, S., Valle, A., Regueiro, B. y Piñeiro, I. (2016). Incidencia de las metas académicas del alumnado de secundaria en su gestión motivacional. Aula Abierta,44(2), 83-90. doi.org/10.1016/j.aula.2016.03.001.

Franco, M. A. (2015). Competencias científicas en la enseñanza y el aprendizaje por investigación. Un estudio de caso sobre corrosión de metales en secundaria. Enseñanza de las Ciencias, 33(2), 231252. doi:http://dx.doi.rg/10-5565/revensciencas1645.

Gaeta, M. L. (2009). La autorregulación del aprendizaje: la estructura del aula, la orientación a metas y las estrategias volitivas y metacognitivas en escolares adolescentes. Revista Electrónica Interuniversitaria de Formación del Profesorado, 12(2), 157.

Gaeta, M. L., Teruel, M. P. y Orejudo, S. (2012). Aspectos motivacionales, volitivos y metacognitivos del aprendizaje autorregulado. Electronic Journal of Research in Educational Psychology, 10(1), 073094. 
Gaeta, M. L., Cavazos, J., Sánchez, A. P., Rosário, P. y Högemann, J. (2015). Propiedades psicométricas de la versión mexicana del Cuestionario para la Evaluación de Metas Académicas (CEMA). Revista Latinoamericana de Psicología, 47(1), 16-24.

García, T. y Pintrich, P. (1993). Intraindividual differences in students' motivation and self-regulated learning. German Journal of Educational Psychology, 7(3), 99-107.

González-Torres, M. C. (2012). Más allá de la motivación: cultivar la voluntad de aprender para hacer frente a las demandas escolares, favorecer el éxito escolar y el desarrollo positivo de los estudiantes. IDEA, 39, 31-94.

González, R., Valle, A., Suárez, J. y Fernández, A. (1999). Un modelo integrador explicativo de la relación entre metas académicas, estrategias de aprendizaje y rendimiento académico. Revista de Investigación Educativa, 17(1), 47-70.

Hayamizu, T. y Weiner, B. (1991). A test of Dweck's model of achievement goals asrelated to perceptions of ability. The Journal of Experimental Education, 59(3), 226-234.

Juvonen, J., Wentzel, K. R. y Gutiérrez, S. N. (2001). Motivación y adaptación escolar: Factores sociales que intervienen en el éxito escolar. México: Oxford University Press.

Kim, C. y Bennekibn, K. N. (2013). Design and implementation of volitional control support in mathematics courses. Education Teach Research Development, 61, 793-817.

Kuhl, J. (1987). Action control: The maintenance of motivational states. In Motivation, intention, and volition (pp. 279-291). Berlin: Springer.

Martín, P. M., Bueno, A. J. y Ramírez, D. M. C. (2010). Evaluación del aprendizaje autorregulado en estudiantes de bachillerato mexicanos. Aula Abierta, 38(1), 59-70.

McCann, C. y García, T. (1999). Maintaining Motivation and Regulating emotion: measuring individual differences in academic volitional strategies. American Educational Research Association, 11(3), 259-279.

McCann, E. J. y Turner, J. E. (2004). Increasing student learning through volitional control. Teachers College Record, 106(9), 1695-1714.

Mega, C., Ronconi, L. y De Beni, R. (2014). Whats makes a good student? How emotions, self-regulated learning, and motivation contribute to academic achievement. Journal of Educational Psychology, 106(1), 121-131.

Midgley, C., Kaplan, A. y Middleton, M. (2001). Performance-Approach Goals: Good for What, for Whom, under What circunstances, and all What Cost? Journal of Educational Psychology, 93 (1), $77-85$.

Navas, L. y Sampascual, M. G. (2008). Un análisis exploratorio y predictivo sobre las orientaciones de meta y sobre el contenido de las metas de los estudiantes. Horizontes Educacionales 13(1), 23-34.

Navas, L., Soriano, L. J., Holgado, F. P. y Jover, I. (2016). Las metas múltiples: Análisis predictivo del rendimiento académico en estudiantes chilenos. Educación XXI, 19(1), 267-285. doi:10.5944/ educXX1.14225 
Núñez, J. C., González-Pienda, J. A., González-Pumariega, S., García, M. y Roces, C. (1997). Cuestionario para la evaluación de metas académicas en Secundaria (CEMA-II). Departamento de Psicología, Universidad de Oviedo.

OCDE (2006). Marco de la evaluación. Conocimientos y habilidades en Ciencia, Matemáticas y Lectura. Programa para la Evaluación Internacional de Alumnos. Santillana Educación para la edición española. www.marcosteoricospisa2006.pdf.

Ormrod, E. J. (2005). Aprendizaje Humano. Madrid: Pearson-Prentice Hall.

Pintrich, P. R. (1999). The role of motivation in promoting and sustaining self-regulated learning. International Journal of Educational Research, 31(6), 459-470.

Pintrich, P. R (2003). A motivational science perspective on the role of student motivation in learning and teaching context. Journal of Educational Psychology, 95(4), 667-686.

Pintrich, P. R. (2000). Multiple Goals, Multiple Pathways: The role of Goal Orientation Learning and Achievement. Journal of Educational Psychology, 92(3), 544-555.

Sanz de Acedo, L., Ugarte, M. D. y Lumbreras, B. M. (2003). Desarrollo y validación de un cuestionario de Metas para Adolescentes. Psicothema, 15(3), 493-499.

Schunk, H. D. y Zimmerman, B. J. (2008). Motivation and Self-Regulated Learning. Theory, Research and Applications. New York: Lawrence Erlbaum Associates.

Valle, A., Cabanach, R., Rodríguez, S., Núñez, J. C. y Gozález-Pienda, J. A. (2006). Metas académicas, estrategias cognitivas y estrategias de autorregulación del estudio. Psicothema, 18(2), 165-170.

Valle, A., Cabanach, R., Rodríguez, S., Núñez, J. C., Gozález-Pienda, J. A. y Rosário, P. (2006). Perfiles motivacionales en estudiantes de secundaria: análisis diferencial en estrategias cognitivas, estrategias de autorregulación y rendimiento académico. Revista Mexicana de Psicología, 26(1), 113-124.

Valle, A., Regueiro, B., Rodríguez, S., Piñeiro, I., Freire, M. y Suárez, N. (2015). Perfiles motivacionales como combinación de expectativas de autoeficacia y metas académicas en estudiantes universitarios. European Journal of Education and Psychology, 8(1), 1-8.

Wentzel, R. K. (2000). What is that I'm trying to achieve? Classroom goals from a content perspective. Contemporary Educational Psychology 25, 105-115.

Recibido: 07-12-2019

Aceptado: 04-05-2020 